\title{
Fasciolosis en Vicuñas (Vicugna vicugna) de la Sierra Central del Perú
}

\author{
Fascioliasis in Vicugna (Vicugna vicugna) in the Central Highlands of Peru \\ Luis Miguel Samamé A. ${ }^{1}$, Amanda Chávez V. ${ }^{1,2}$, Rosa Pinedo V. ${ }^{1}$
}

\section{Resumen}

\begin{abstract}
El objetivo del presente estudio fue determinar la presencia de huevos de Fasciola hepatica en vicuñas silvestres presentes en el cerro Pumacocha del distrito de Paccha, Junín, Perú, así como determinar la asociación con las variables sexo y estrato etario. Se recolectaron 143 muestras de heces tomadas directamente del recto. Las vicuñas fueron capturadas mediante un chaccu realizado por la comunidad campesina local durante la época de esquila anual (setiembre). Los huevos fueron analizados mediante la técnica de sedimentación rápida y la determinación de la carga parasitaria - huevos por gramo de heces (hpg) - mediante el método de McMaster modificado. La frecuencia total de $F$. hepatica fue de $32.9 \%$, sin diferencia estadística por efecto del sexo o grupo etario. La carga promedio fue de $23.7 \mathrm{hpg}$.
\end{abstract}

Palabras clave: Fasciola hepatica, camélidos sudamericanos, McMaster modificado, distomatosis, Junín

\section{Abstract}

The aim of this study was to determine the presence of $F$. hepatica eggs in vicugnas in the Paccha area, Junín, Peru, and to evaluate its association with sex and age. Samples $(\mathrm{n}=143)$ were collected from the rectum during a «chaccu» activity (annual capture of wild vicugnas by the local peasants for shearing (September). Samples were analyzed by the rapid sedimentation technique and the determination of parasite load - eggs per gram

${ }^{1}$ Laboratorio de Microbiología y Parasitología Veterinaria, Facultad de Medicina Veterinaria, Universidad Nacional Mayor de San Marcos, Lima, Perú

2 E-mail: achavezvg@gmail.com

Recibido: 24 de marzo de 2015

Aceptado para publicación: 9 de setiembre de 2015 
of feces (epg) - by the McMaster method. The frequency of $F$. hepatica was $32.9 \%$ and without statistical difference due to sex or age. The average load was $23.7 \mathrm{epg}$.

Key words: Fasciola hepatica, South American camelids, McMaster, distomatosis, Junin

\section{INTRODUCCIÓN}

La fasciolosis o distomatosis es causada por el trematodo Fasciola hepatica, considerada una de las enfermedades parasitarias más importantes en la ganadería del Perú, ocasionando severas pérdidas económicas, estimándose en al menos US $\$ 50$ millones por año. Esto es debido a una baja ganancia de peso, reducida fertilidad y mayor mortalidad y abortos. Asimismo, por el decomiso de vísceras infectadas y los costos de tratamiento; además de los problemas de salud pública que ocasiona (Espinoza et al., 2010).

El parásito se desarrolla en el hígado de una amplia variedad de hospedadores definitivos domésticos y silvestres; desde poligástricos como bovinos, ovinos, caprinos, camélidos sudamericanos (CSA) y venados, a monogástricos como equinos, caninos, cuyes, conejos, vizcachas, e inclusive al hombre, que actúa como hospedador accidental (Sapmaz et al., 2013). La biología de $F$. hepatica implica un ciclo biológico heteroxeno, requiriendo para ello un hospedero definitivo y un hospedero intermediario (caracol de la familia Lymnaeidae). Los parásitos adultos invaden el hígado, donde depositan sus huevos, los cuales son eliminados junto con las heces. En el medio ambiente buscan desarrollarse dentro del caracol y liberarse en el ambiente húmedo como cercaria, donde se enquistará en las pasturas. Los animales, al ingerir el pasto contaminado, se verán afectados, primero con las formas juveniles y luego por las formas adultas que se localizarán en los conductos biliares (Cordero del Campillo et al., 1999).
En general, F. hepatica afecta a los animales de regiones con lluvias moderadas a intensas, aunque también aparece en regiones más secas con valles pantanosos y a lo largo de arroyos o canales de riego que cobijan al caracol intermediario. Las características epidemiológicas requeridas para completar su ciclo biológico y transmisión de la enfermedad son diversas, complejas y únicas: adecuada temperatura ambiental, humedad, numerosos reservorios de agua, viabilidad del hospedero intermediario, hábitos dietéticos y animales infectados, principalmente ganado ovino y bovino (Olaechea, 2007).

La infección por $F$. hepatica ha sido descrita con alguna frecuencia en sus distintas formas (aguda, subaguda y crónica) en camélidos sudamericanos domésticos (Leguía, 1999; Cafrune et al., 2004); en cambio, los registros de esta parasitosis en camélidos silvestres son escasos. Se dispone de los estudios de Issia et al. (2007) en guanacos (Lama guanicoe) silvestres y los de Cafrune et al. (2004) en vicuñas (Vicugna vicugna) y Olaechea y Abad (2005) en guanacos, ambos con un tipo de crianza de semi-cautividad.

El Perú ha llegado a ser el mayor productor de fibra de vicuña a nivel mundial, comercializando hasta $5500 \mathrm{~kg}$ por año, lo que genera un sustancial ingreso económico a las comunidades altoandinas (Quispe et al., 2009). Sin embargo, el contacto de las vicuñas con el ganado doméstico conlleva a posibles transmisiones de parásitos gastrointestinales entre especies ganaderas, comprometiendo seriamente su supervivencia. Por ello, el objetivo del presente estudio fue determi- 
nar la presencia de huevos de $F$. hepatica en las heces de vicuñas es una zona de crianza en la zona central del país, y evaluar su asociación con la edad y sexo.

\section{Materiales y Métodos}

El presente estudio se realizó en el cerro Pumacocha, perteneciente al distrito de Paccha, ubicado en la parte centro-oriental de la Provincia de Yauli (11 '28' $12^{\prime \prime}$ latitud sur, $75^{\circ} 57^{\prime} 32^{\prime \prime}$ latitud oeste, $3742 \mathrm{msnm}$ ), durante la primera semana de setiembre de 2010 . El procesamiento de la muestras de heces se llevó a cabo en el Laboratorio de Parasitología de la Facultad de Medicina Veterinaria (FMV) de la Universidad Nacional Mayor de San Marcos (UNMSM), Lima.

La especie en estudio, al ser un animal silvestre, fue trabajada durante la época de esquila, donde se realiza un atrape de estos animales denominado Chaccu o «rodeo» (Tuppia, 2009), técnica ancestral de arreo que permite la captura de animales vivos, a cargo de personal especializado. La esquila tarda poco más de dos minutos por animal, incluyendo en este caso, la toma de la muestra de heces.

Se colectaron aproximadamente $15 \mathrm{~g}$ de heces por animal utilizando bolsas de plástico y tomadas directamente del recto de la totalidad de las vicuñas capturadas $(\mathrm{n}=143)$. Se registró el sexo y estrato etario (cría [ $<1$ año], juvenil [1-2 años], adulto [3 años]). El material fecal fue almacenado en recipientes térmicos con refrigerante para su traslado y posterior análisis.

Para la evaluación coproparasitológica se utilizó la técnica de sedimentación rápida de Lumbreras (TSRL) (Lumbreras et al., 1962), a fin de identificar muestras positivas a Fasciola. Asimismo, la carga parasitaria, es decir, el número de huevos de $F$. hepatica por gramo de heces (hpg), se determinó me- diante la técnica de Conceição et al. (2002), pero con el uso de tamices (Girão y Ueno, 1985).

Se estimó la frecuencia relativa porcentual de la enfermedad, en base al número de muestras positivas obtenidas (Daniel, 2004), y se expresó en promedio e intervalo de confianza al 95\%. Se determinó la asociación de las variables grupo etario y sexo con la presencia de la enfermedad mediante la prueba de Chi cuadrado. Se contemplaron las normas éticas para la investigación señaladas por el Comité de Ética de la FMV-UNMSM para Uso de Animales de Experimentación.

Adicionalmente, se realizó la evaluación morfológica de los huevos de $F$. hepatica encontrados en las heces de las vicuñas, determinando la forma (huevos redondos: largo/ancho $=1$; huevos elípticos: largo/ancho $>1$ ) y el tamaño (multiplicando las medidas del largo por el ancho), según Abrous et al. (1998).

\section{Resultados}

La frecuencia de vicuñas silvestres con presencia de huevos de $F$. hepatica en heces fue de $32.9 \pm 7.7 \%$, donde la frecuencia fue ligeramente mayor en machos (35.8\%) que en hembras (29\%) y las vicuñas juveniles presentaron la frecuencia más alta (45.7\%). No obstante, no se encontró diferencia estadística por efecto del sexo o estrato etario (Cuadro 1).

El promedio geométrico de la carga parasitaria de F. hepatica fue de 23.7 huevos por gramos de heces (hpg); sin diferencia estadística por efecto de las variables sexo y grupo etario (Cuadro 2). Adicionalmente, el resultado de las 100 determinaciones de las medidas biométricas del huevo de este parásito en vicuñas resultó en un promedio de 72.7 $\pm 8.2 \mu \mathrm{m}$ de ancho por $127.8 \pm 15.0 \mu \mathrm{m}$ de largo (Fig. 1). 


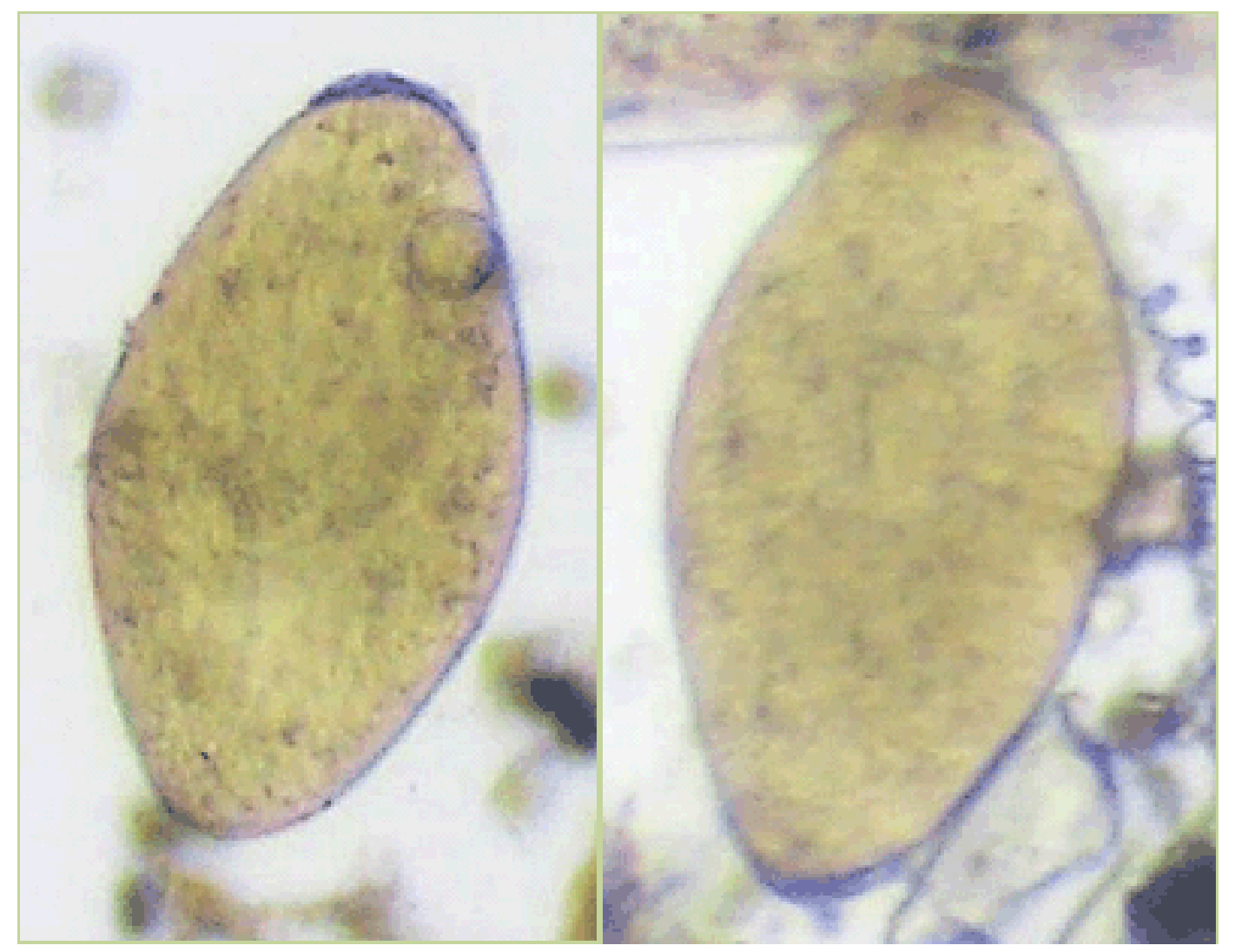

Figura 1. Huevos de Fasciola hepatica hallados en heces de vicuñas en el distrito de Paccha, provincia de Yauli, Junín (2010)

Cuadro 1. Frecuencia de Fasciola hepatica en vicuñas según estrato etario y sexo en el distrito de Paccha, Yauli, Junín (2010)

\begin{tabular}{lcc}
\hline Variable & $\begin{array}{c}\text { Animales } \\
(\mathrm{n})\end{array}$ & $\begin{array}{c}\text { Positivos } \\
(\%)\end{array}$ \\
\hline Grupo etario & & \\
$\quad$ Crías & 18 & 5.6 \\
Juveniles & 35 & 45.7 \\
$\quad$ Adultos & 90 & 33.3 \\
Sexo & & \\
$\quad$ Machos & 81 & 35.8 \\
$\quad$ Hembras & 62 & 20.0 \\
\hline Total & 143 & $32.9 \pm 7.7$ \\
\hline
\end{tabular}

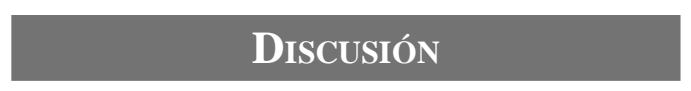

En la década del ochenta, diversos investigadores cuestionaban la presencia de $F$. hepatica en alturas superiores a los 4000 msnm (región Jalca o Puna), debido a las variaciones drásticas de temperatura diurnanocturna, llamadas comúnmente «heladas», que determinan un ambiente seco y árido y, por lo tanto, desfavorables para el desarrollo del parásito (Leguía, 1991). Sin embargo, Londoñe et al. (2009) demostraron que, debido al calentamiento global, las condiciones ambientales a esas altitudes habían variado, pudiendo encontrarse especies de caracoles albergando formas larvarias de distoma hepático en altitudes superiores a los $4000 \mathrm{~m}$. 
Cuadro 2. Carga promedio de huevos de Fasciola hepatica (hpg) en vicuñas según estrato etario y sexo en el distrito de Paccha, provincia de Yauli, Junín (2010)

\begin{tabular}{lccc}
\hline Variable & $\begin{array}{c}\text { Positivos } \\
(\mathrm{n})\end{array}$ & $\begin{array}{c}\mathrm{PG}^{1} \\
(\mathrm{hpg})\end{array}$ & Rangos \\
\hline $\begin{array}{c}\text { Grupo etario } \\
\text { Crías }\end{array}$ & 1 & 25.0 & 25 \\
$\quad$ Juveniles & 16 & 29.4 & $(8-375)$ \\
$\quad$ Adultos & 30 & 23.0 & $(8-861)$ \\
Sexo & & & \\
$\quad$ Machos & 29 & 25.9 & $(8-861)$ \\
$\quad$ Hembras & 18 & 20.0 & $(8-158)$ \\
\hline Total & 47 & 23.7 & $(8-861)$ \\
\hline${ }^{1}$ Promedio geométrico & &
\end{tabular}

Los resultados del presente estudio confirman la presencia de $F$. hepatica en vicuñas silvestres que habitan el cerro Pumacocha, a una altitud de 3800 a $4500 \mathrm{msnm}$ del distrito de Paccha, en la sierra central del país, y con una frecuencia de $32.9 \%$. Los estudios de Cafrune et al. (2004) en vicuñas silvestres criadas en semicautividad y los de Olaechea y Abad (2005) en guanacos repor$\tan$ frecuencias de 8 a $26 \%$ y $10 \%$, respectivamente, mientras que Issia et al. (2007) hallaron $14 \%$ de prevalencia en guanacos silvestres de Argentina, valores inferiores al $32.9 \%$ del presente estudio.

La elevada frecuencia encontrada podría ser explicada por el hecho que la vicuña se encuentra compartiendo su hábitat con animales domésticos llevados por el hombre, como ovejas, CSA domésticos (alpacas y llamas) y equinos (caballos y mulas). Esta característica de pastoreo conjunto fue evidenciada al momento del chaccu, donde se pudo observar heces de otros animales en las áreas de captura. Además, se conoce que grupos de vicuñas suelen pastear con tropas de alpacas en horas de la mañana (Michaud, 2009).
Se conoce por estudios anteriores que ovinos (Ticona et al., 2010) y CSA de la sierra central presentan grados moderados y altos de infección por distoma hepático. Recientemente, se hallaron prevalencias de $49.5 \mathrm{y}$ $73.8 \%$ en llamas y alpacas, respectivamente, en empresas ganaderas de Junín (Flores et al., 2014). Estos animales son considerados como importantes reservorios de $F$. hepatica al cumplir un rol epidemiológico en la transmisión y diseminación de la fasciolosis, debido a la dispersión de huevos de distoma en las heces. Además, la alta resistencia de los huevos del parásito a las bajas temperaturas $\left(-16{ }^{\circ} \mathrm{C}\right)$ por periodos prolongados, les permite continuar su desarrollo en el momento que se presentan condiciones ambientales favorables (Cordero del Campillo et al., 1999; González, 1999; Barriga, 2002; Carrada, 2007), permitiendo una infección cruzada con las vicuñas presentes en la zona.

Es probable que la frecuencia real de esta parasitosis pueda estar subestimada, ya que la evaluación se realizó mediante exámenes coproparasitológicos, cuya sensibilidad varía entre 33 a $92 \%$ cuando se procesan muestras de heces de 3 a $30 \mathrm{~g}$ (Meissonnier y Mage, 2007). La sensibilidad de estas pruebas podrían incrementarse con toma seriada de muestras (Rapsch et al. 2006), aspecto que no podría hacerse en este caso debido a la dificultad en el manejo de la vicuña, cuya captura es realizada una vez al año, y donde solo se pudo extraer cerca de $15 \mathrm{~g}$ de heces por animal.

Existen métodos de diagnóstico más sensibles y costosos como un ELISA indirecto para la detección de Ac y otro de coproantígeno para la detección de antígenos de secreción y excreción para $F$. hepatica en alpacas (Li et al., 2005), con sensibilidad y especificidad del $100 \%$ para camélidos sudamericanos. Además, existe una técnica de ELISA para la detección de anticuerpos circulantes contra antígenos Fas2 (Neyra et al., 2002) con sensibilidad y especificidad del $95 \%$, pero lamentablemente, estas técnicas no son de uso comercial. 
No se encontraron diferencias por efecto del sexo debido a que tanto el macho como la hembra están expuestos a similares condiciones de pastoreo y, por lo tanto, sometidos a los mismos riesgos de infección. Las características de convivencia entre machos y hembras están ligadas al comportamiento social de la vicuña, las cuales se organizan por grupos familiares conformados, usualmente, por un macho territorial con 3 a 4 hembras y ubicados en terrenos estables, generalmente permanentes durante todo el año (Vilá, 2000).

Si bien no se halló diferencia estadística por estrato etario en la frecuencia de fasciolosis, se observó que la infección fue más alta en los juveniles. Las vicuñas juveniles forman grupos migratorios (tropillas de solteros) que no poseen terrenos establecidos y caminan de manera errante, desplazándose muchos kilómetros en pocos días en busca de terrenos donde alimentarse (Michaud, 2009); de esta manera requieren de más energía por lo que necesitan mayor consumo de forraje, presentando mayor posibilidad de exposición a la infección. Además, es conocido que los animales jóvenes son los más afectados en comparación a los adultos (Cordero del Campillo et al., 1999).

La cuantificación de los huevos de Fasciola hepatica tiene el objetivo de señalar el riesgo al que están expuestos los animales. No existe este tipo de estudios en camélidos sudamericanos, de allí que el 23.7 de promedio geométrico de la carga de los huevos, con rangos de 8 a 601 puede ser contrastado con la información existente en ovinos. En esa especie, en los grados de infestación se considera una carga de 50 a $<200$ como leve, de 200 a 500 como intermedio y $>500$ hpg como infección grave (Soulsby, 1993; Kassai, 2002). Es así, que los resultados obtenidos podrían ser considerados como infecciones leves. Flores et al. (2014) hallaron cargas de 12.6 y 19.9 hpg en llamas y alpacas, respectivamente.
Al comparar el tamaño de los huevos de $F$. hepatica en las vicuñas del estudio, se pudo determinar que son de mayor tamaño que los de llamas y alpacas. Además, son más elípticos que los de la llama (Flores et al., 2014), lo cual es un indicativo que las especies hospedadores definitivas influyen en el tamaño de los huevos y de la forma adulta de la F. hepatica (Valero et al., 2001).

\section{Conclusiones}

- La frecuencia de huevos de F. hepatica hallada en heces de vicuñas en el distrito de Paccha, provincia de Yauli, Junín, fue de $32.9 \pm 7.7 \%$ (47/143).

- No se halló diferencia estadística entre la frecuencia de $F$. hepatica con las variables sexo y estrato etario.

- La carga parasitaria promedio de huevos de F. hepatica fue de $23.7 \mathrm{hpg}$.

\section{Literatura Citada}

1. Abrous M, Comes AM, Gasnier N, Rondelaud D, Dreyfuss G, Chauvin A, Ménard, et al. 1998. Morphological variability in $F$. hepatica eggs in ruminants, rodents and lagomorphs. $\mathrm{J}$ Helminthol 72: 313-317. doi: 10.1017/ S0022149X00016667

2. Barriga O. 2002. Las enfermedades parasitarias de los animales domésticos de América Latina. $2^{\mathrm{a}}$ ed. Santiago de Chile: Germinal. 247 p.

3. Cafrune MM, Aguirre DH, Freytes I. 2004. Fasciolosis en vicuñas (Vicugna vicugna) en semi-cautiverio de Molinos, Salta, Argentina, con notas de otros helmintos en este hospedador. Vet Arg 21:513-520.

4. Carrada BT. 2007. Fasciola hepatica: Ciclo biológico y potencial biótico. Rev Mex Patol Clin 54(1): 21-27.

5. Conceição M, Durão R. CI, Costa I, Correia da Costa J. 2002. Evaluation of a simple sedimentation method 
(modified McMaster) for diagnosis of bovine fascioliosis. Vet Parasitol 105: 337-343.

6. Cordero del Campillo M, RojoVásquez FD, Martínez AR, Sánchez MC, Hernández S, Navarrete I, Diez $\boldsymbol{P}$, et al. 1999. Parasitología veterinaria. España: Mc-Graw Hill Interamericana. 968 p.

7. Daniel WM. 2004. Bioestadística: Base para el análisis de las ciencias de la $\mathrm{Sa}$ lud. 4a ed. México: Ed Limusa. 203 p.

8. Espinoza JR, Terashima A, HerreraVelit P, Marcos LA. 2010. Fasciolosis humana y animal en el Perú: impacto en la economía de las zonas endémicas. Rev Perú Med Exp Salud Públ 27: 604-612. doi: 10.1590/S1726-46342010000400018

9. Flores B, Pinedo $R V$, Suarez $F$, Angelats $R$, Chávez V. 2014. Prevalencia de fasciolosis en llamas y alpacas en dos comunidades rurales de Jauja, Perú. Rev Inv Vet Perú 25: 284-292. doi: 10.15381/rivep.v25i2.8501

10 Girão ES, Ueno H. 1985. Diagnóstico coprológico quantitativo da fasciolose de ruminantes no Rio Grande do Sul. Pesq. Agropec Bras 20: 461-466.

11. González GM. 1999. Incidencia de Fasciola hepatica en la cabaña ganadera asturiana. Ind Lac Esp 249: 50-52.

12. Issia L, Ovejero R, Carmanchahi P, Pietrokovsky S, Wisnivesky-Colli $C$. 2007. Primer registro de $F$. hepatica en guanacos silvestre de Mendoza, Argentina. En: V Congreso Latinoam Especialistas en Pequeños Rumiantes y CSA. Buenos Aires.

13. Kassai T. 2002. Helmintología veterinaria. Zaragoza: Acribia. 296 p.

14. Leguía G. 1991. Distomatosis hepática en el Perú. Epidemiología y control. $2^{a}$ ed. Lima: Univ Nacional Mayor de San Marcos. 45 p.

15. Leguía G. 1999. Enfermedades parasitarias de camélidos sudamericanos. Lima: Del Mar. 190 p.

16. Li O, Leguía G Espino A, Duménigo B, Díaz A, Otero O. 2005. Detección de anticuerpos y antígenos para el diagnóstico de $F$. hepatica en alpacas naturalmente infectadas. Rev Inv Vet Perú 16: 143-153. doi: 10.15381/rivep. v16i2.1554

17. Londoñe P, Chávez A, Li O, Suárez F, Pezo D. 2009. Presencia de caracoles Lymnaeidae con formas larvarias de Fasciola hepatica en altitudes sobre los 4000 msnm en la sierra sur del Perú. Rev Inv Vet Perú 20: 58-65. doi: 10.15381/ rivep.v20i1.533

18. Lumbreras H, Cantella $R$, Burga $R$. 1962. Acerca de un procedimiento de sedimentación rápida para investigar huevos de Fasciola hepatica en las heces, su evaluación y uso en el campo. Rev Med Peruana 31: 167-174.

19. Meissonnier E, Mage C. 2007. Les méthodes de détection de Fasciola hepatica dans les troupeaux bovins en France. Bull Acad Vét 160: 395-406.

20. Michaud C. 2009. La vicuña (Vicugna vicugna mensalis) en el Perú: historia, biología de la especie, sistemas de manejo actual y su relación con el bienestar animal. Tesis de Médico Veterinario. Lima: Univ Nacional Mayor de San Marcos. 47 p.

21. Neyra V, Chavarry E, Espinoza JR. 2002. Cysteine proteinases Fas1 and Fas2 are diagnostic markers for $F$. hepatica infection in alpacas (Lama pacos). Vet Parasitol 105: 21-32. doi: 10.1016/S0304-4017(02)00002-X

22. Olaechea FV, Abad M. 2005. An outbreak of fascioliasis in semicaptive guanacos (Lama guanicoe) in Patagonia (Argentina). First report. En: XX International Conference of the World Association for the Advancement of Veterinary Parasitology. Christchurch, New Zealand.

23. Olaechea FV. 2007. Fasciola hepatica. En: Suárez VH, Olaechea FV, Romero JR, Rossanigo CE (eds). Enfermedades parasitarias de los ovinos y otros rumiantes menores en el cono sur de América. Argentina: INTA. p 159-168. 
24. Quispe EC, Rodríguez TC, Iñiguez LR, Mueller JP. 2009. Producción de fibra de alpaca, llama, vicuña y guanaco en Sudamérica. Anim Genetic Resources 45: 1-14.

25. Rapsch C, Schweitzer G, Grimm F, Kohler L, Bauer C, Deplazes P, Braun $U$, Togerson PR. 2006. Estimating the true prevalence of Fasciola hepatica in cattle slaughtered in Switzerland in the absence of an absolute diagnostic test. Int J Parasitol 36: 1153-1158. doi: 10.1016/j.ijpara.2006.06.001

26. Sapmaz F, Kalkan IH, Guliter S, Nazlýoðlu A. 2013. Aclinical presentation of a very rare infection: parenchymal Fasciola hepatica. Turkiye Parazitol Derg 37: 305-306. doi: 10.5152/tpd.2013.2998

27. Soulsby EJ. 1993. Parasitología y enfermedades parasitarias en los animales domésticos. $7^{\text {a }}$ ed. México: Interamericana. $823 \mathrm{p}$.
28. Ticona D, Chávez A, Casas E, Chavera A, Li Olga. 2010. Prevalencia de Fasciola hepatica en bovinos y ovinos de Vilcashuamán. Ayacucho. Rev Inv Vet Perú 21: 168-174.

29. Tuppia M. 2009. Manejo sustentable de la vicuña con inclusión social, económica, ambiental. Lima, Perú: Dirección General Forestal y Fauna Silvestre, Ministerio de Agricultura. $27 \mathrm{p}$.

30. Valero M, Darce NA, Panova M, MasComa S. 2001. Relationships between host species and morphometric patterns in F. hepatica adults and eggs from the northern Bolivian Altiplano hyperendemic región. Vet Parasitol 102: 85-100.

31. Vilá BL. 2000. Comportamiento y organización social de la vicuña. En: Gonzales B, Bas F, Tala C, Iriarte A(eds). Manejo Sustentable de la vicuña y el guanaco. Chile: Pontificia Universidad Católica de Chile. p 175-192 